\title{
Supplement Materials for the Salt-gradient Approach for Regulating Capture-to-Translocation Dynamics of DNA with Nanochannel Sensors
}

\author{
Yuhui He ${ }^{1}$, Makusu Tsutsui ${ }^{* 2}$, Ralph H. Scheicher ${ }^{3}$, Xiang Shui \\ Miao ${ }^{\dagger 1}$, and Masateru Taniguchi ${ }^{2}$ \\ ${ }^{1}$ School of Optical and Electronic Information, Huazhong \\ University of Science and Technology, LuoYu Road, Wuhan \\ 430074, China \\ ${ }^{2}$ The Institute of Scientific and Industrial Research, Osaka \\ University, 8-1 Mihogaoka, Ibaraki, Osaka 567-0047, Japan \\ ${ }^{3}$ Division of Materials Theory, Department of Physics and \\ Astronomy, Angström Laboratory, Uppsala University, Box 516, \\ SE-751 20, Uppsala, Sweden
}

May 13, 2016

\begin{abstract}
The supplement materials include detailed derivations of Poisson-Boltzmann equation along nanochannel radial direction, analytic expression for salt gradient in the nanochannel, Teorell-Meyer-Sievers model for monovalent and divalent solution, $\Delta V_{\sigma}$ and $\Delta V_{D}$ for monovalent and divalent ions, equation for nanofluidics under salt gradient, DNA capture by electrical field and exclusive electrical field in II region.
\end{abstract}

*makusu32@sanken.osaka-u.ac.jp

† miaoxs@hust.edu.cn 


\section{Contents}

S1 Poisson-Boltzmann equation for channel radial direction $\quad$ S2

S2 Salt gradient in nanochannel

S3 Teorell-Meyer-Sievers model for monovalent and divalent solution

S4 Derivation of $V_{\sigma}$ and $V_{D}$ for monovalent and divalent ions

S5 Equation for nanofluidics under salt gradient $\quad$ S7

S5.1 Simplified view . . . . . . . . . . . . . . . S7

S5.2 Strict derivation . . . . . . . . . . . . . . S7

S6 The role of diffusio-osmosis $\quad$ S8

S7 DNA capture by electrical field $\quad$ S9

S8 Exclusive electrical field in II region $\quad$ S9

S9 Fitting LiCl concentration gradient experiments $\quad$ S9

\section{S1 Poisson-Boltzmann equation for channel ra- dial direction}

The channel-radial component of the ionic flux is composed by electrophoretic migration, diffusion and convection as follows:

$$
\vec{j}_{i} \cdot \vec{e}_{r}=-\operatorname{sgn}\left(z_{i}\right) n_{i} \mu_{i} \frac{\partial V}{\partial r}-D_{i} \frac{\partial n_{i}}{\partial r}+\vec{u} \cdot \vec{e}_{r}=0
$$

In the above, the subscript $i$ represents the $i^{t h}$ type of ions. The sgn funtion means the sign of the ion valence. Other parameters are the same as those defined in the main context. Due to the restriction by the channel wall, the radial flux of ions should be zero.

Besides, the fluidic speed along the radial direction should also be zero due to the wall:

$$
\vec{u} \cdot \vec{e}_{r}=0
$$

By utilizing the Einstein relation, we arrive at the following expression for the ion distribution from the above two equations:

$$
n_{i}(r, z)=n_{i}\left(r_{0}, z\right) \exp \left(-\frac{z_{i} e \phi(r, z)}{k T}\right)
$$


where $\phi(r, z)=V(r, z)-V\left(r_{0}, z\right)$. On the other hand, the electrostatic equation reads as follows

$$
\frac{1}{r} \frac{\partial}{\partial r}\left(r \frac{\partial \phi(r, z)}{\partial r}\right)=-\frac{\Sigma_{i} z_{i} e C_{0} \exp \left(-z_{i} e \phi(r, z) / k T\right)}{\varepsilon}
$$

By combining the above two equations, the Poisson-Boltzmann distribution shown in the main context is arrived at. Besides, for the $\mathrm{MgCl}_{2}$ and $\mathrm{KCl}$ salt in nanoslit system used in the experiments [3] the Poisson-Boltzmann equation is rewritten in Descartes coordinate as follows:

$$
\frac{\partial^{2}}{\partial y^{2}} \phi(y, z)=-\frac{\Sigma_{i} z_{i} e C_{0} \exp \left(-z_{i} e \phi(y, z) / k T\right)}{\varepsilon}
$$

\section{S2 Salt gradient in nanochannel}

As seen in Fig.S1 the cis/trans chamber is divided into two regions, I and II. The imposed salt concentration difference $\left(C_{\max }-C_{\min }\right)$ will be dropped in I and II regions of the two chambers and the inside of the channel. There have been analytic expressions for salt distribution within I regions of chambers and the inside of channel: the former is an inversion-to-radius relation required by the ion flow continuity while the latter is approximated with linear drop. Nonetheless, the challenge is to evaluate the salt gradient within the channel entrance/exit region as marked by II in the figure.

Here we make use of the mathematical similarity between salt difference and voltage difference. Assume that the voltage drop inside the nanochannel (here it is a traditionally homogeneous sanity system with concentration $\left.n_{0}\right)$ is $(\Delta V)_{p}$. The forwarding flow of ions through the nanochannel is

$$
I=n_{0} \Sigma_{i} \mu_{i} \frac{(\Delta V)_{p}}{L} \pi R^{2}
$$

Considering the continuity of ion flow, the electrical field in I region of cis chamber should show an inverse square dependence on the distance from the channel mouth $r$

$$
E(r)=\frac{I}{n_{0} \Sigma_{i} \mu_{i} 2 \pi r^{2}}
$$

The voltage within I region is then arrived at according to $-\partial V / \partial r=E(r)$ :

$$
V(r)=-(\Delta V)_{p} \frac{R^{2}}{2 L r}
$$

so that the voltage drop $(\Delta V)_{\text {I }}$ within I region of cis chamber is

$$
(\Delta V)_{\mathrm{I}}=(\Delta V)_{p} \frac{R}{2 L}
$$

On the other hand, the resistances of I, II region and nanopore inside have been shown as follows [2]

$$
R_{\mathrm{I}}: R_{\mathrm{II}}: R_{p}=\frac{1}{2 \pi R}:\left(\frac{1}{4 R}-\frac{1}{2 \pi R}\right): \frac{L}{\pi R^{2}}
$$


Therefore, the corresponding voltage drop should be

$$
(\Delta V)_{\mathrm{I}}:(\Delta V)_{\mathrm{II}}:(\Delta V)_{p}=\frac{1}{2}:\left(\frac{\pi}{4}-\frac{1}{2}\right): \frac{L}{R}
$$

Following quite similar derivations, we obtain the relation between salt concentration drop within I region and that inside the nanochannel:

$$
(\Delta C)_{\mathrm{I}}:(\Delta C)_{p}=\frac{R}{2 L}
$$

By taking the previously derived voltage drop as a reference, we find the salt concentration bias within each region as follows:

$$
(\Delta C)_{\mathrm{I}}:(\Delta C)_{\mathrm{II}}:(\Delta C)_{p}=\frac{1}{2}:\left(\frac{\pi}{4}-\frac{1}{2}\right): \frac{L}{R}
$$

while the sum of $2(\Delta C)_{\mathrm{I}}, 2(\Delta C)_{\mathrm{II}}$ and $(\Delta C)_{p}$ is $\left(C_{\max }-C_{\min }\right)$. Therefore we conclude that the salt gradient within the channel is

$$
-\frac{\partial C}{\partial z}=\frac{(\Delta C)_{p}}{L}=\frac{C_{\max }-C_{\min }}{L+\pi R / 2}
$$

Furthermore, the expression for the salt concentration along the channel axis is

$$
C_{0}(z)=-\frac{C_{\max }-C_{\min }}{L+\pi R / 2} z+\left(C_{\max }+C_{\min }\right) / 2
$$

where the channel axis is located along $-L / 2 \leq z \leq L / 2$.

In I region of left chamber, the salt concentration along axial direction is

$$
C_{0}(z)=C_{\max }-\frac{R}{-(z+L / 2)}(\Delta C)_{\mathrm{I}} \quad(z<-L / 2-R)
$$

where $(\Delta C)_{\mathrm{I}}$ is derived from Eq.S13 as follows

$$
(\Delta C)_{\mathrm{I}}=\frac{R / 2}{L+\pi R / 2}\left(C_{\max }-C_{\min }\right)
$$

In I region of the right chamber, the salt concentration along axial direction is

$$
C_{0}(z)=C_{\min }+\frac{R}{z-L / 2}(\Delta C)_{\mathrm{I}} \quad(z>L / 2+R)
$$

\section{S3 Teorell-Meyer-Sievers model for monovalent and divalent solution}

Here we derive the expression of self-built potential $\Delta V$ according to the TeorellMeyer-Sievers (TMS) models [4, 5, 8]. For monovalent ions, the TMS model is based on the following two assumptions of ion distribution:

$$
\bar{C}_{+}(z)-\bar{C}_{-}(z)=-2 \frac{\sigma_{w}}{N_{A} e R}
$$




$$
\bar{C}_{+}(z) \bar{C}_{-}(z)=C_{0}^{2}(z)
$$

In the above $\bar{C}_{+}$and $\bar{C}_{-}$are the equivalent concentrations of monovalent cations and anions along channel axial direction. That is, $\bar{C}_{ \pm}$is assumed to have been averaged over the channel-radial direction. $C_{0}$ is the concentration of imposed salt along channel axis $z$ as shown in Eq.S15, and $N_{A}$ is the Avogadro Number. Note that in the above expressions the salt concentrations $\bar{C}_{ \pm}$are in unit of $\mathrm{mol} / \mathrm{m}^{3}$.

The assumptions shown in Eq.S19 and S20 capture two major items of the physical mechanisms: first, the surface charges on the channel wall have to be equilibrated by the excess counterions in the solution; second, the Boltzmann distribution of cations and anions holds along channel radial direction so that $C_{+}(r) C_{-}(r)=C_{0}\left(r_{0}\right) \exp [-e \phi(r) / k T] C_{0}\left(r_{0}\right) \exp [e \phi(r) / k T]=C_{0}^{2}$. Then the self-built local electrical field is estimated by

$$
E_{z}=\frac{t_{+}-t_{-}}{t_{+}+t_{-}} \frac{k T}{e} \frac{\partial \ln C_{0}}{\partial z}
$$

where $t_{ \pm}$is the transport number of the cations/anions:

$$
t_{ \pm}=\frac{D_{ \pm} \bar{C}_{ \pm}}{D_{+} \bar{C}_{+}+D_{-} \bar{C}_{-}}
$$

However, for the $\mathrm{MgCl}_{2}$ solution in a nanoslit system [3] the above assumptions have to be modified as follows:

$$
\begin{gathered}
2 \bar{C}_{+}(z)-\bar{C}_{-}(z)=-\frac{2 \sigma_{w}}{N_{A} e W_{y}} \\
\bar{C}_{+}(z) \bar{C}_{-}^{2}(z)=C_{0}(z) \exp \left[-\frac{2 e \phi(r, z)}{k T}\right]\left[2 C_{0}(z) \exp \left(\frac{e \phi(r, z)}{k T}\right)\right]^{2}=4 C_{0}^{3}(z) \\
\frac{\partial V_{0}}{\partial z}=-E_{z}=-\frac{D_{+} \bar{C}_{+}-D_{-} \bar{C}_{-}}{2 D_{+} \bar{C}_{+}+D_{-} \bar{C}_{-}} \frac{k T}{e} \frac{\partial \ln C_{0}}{\partial z}
\end{gathered}
$$

In the above, $W_{y}$ is the width of the nanoslit $(\sim 20 \mathrm{~nm})$ while $\bar{C}_{ \pm}$are ion concentrations averaged over half width $\left(W_{y} / 2\right)$.

The calculation results are plotted in Figs.S2 and S3 with dash lines while those by our electrokinetic model are with real lines. It demonstrates that the TMS model and ours are largely equivalent. Yet at small salt concentrations the results deviate to some extent. From the above equations we are aware that the deviation is caused by the assumption of concentration average over the radial direction (Eq.S20 and Eq.S24). Given small concentration of imposed salt, the thickness of EDL would become sufficiently large and consequently the radial variation of the ion distribution is no longer negligible. Moreover, as we discuss in the main context, the observation IV is caused by the radial variation of net charges. Since that variation is neglected in Eq.S20, TMS model is insufficient to reproduce the related phenomenon. 


\section{S4 Derivation of $V_{\sigma}$ and $V_{D}$ for monovalent and divalent ions}

For monovalent ion system, we start from the zero electrical current equation for the open-circuit measurement

$$
\left(-\mu_{+} \frac{\partial V}{\partial z} \Lambda_{+}-D_{+} \frac{\partial \Lambda_{+}}{\partial z}\right)-\left(\mu_{-} \frac{\partial V}{\partial z} \Lambda_{-}-D_{-} \frac{\partial \Lambda_{-}}{\partial z}\right)=0
$$

where $V$ is composed by the electromotive part and the electrostatic part $V=$ $V_{0}(z)+\phi(r, z)$. By noticing the definition

$$
\Lambda_{ \pm}=C_{0} \exp \left(\mp \frac{e \phi(r, z)}{k T}\right)
$$

we attain the following relation

$$
\frac{\partial \Lambda_{ \pm}}{\partial z}=\frac{\Lambda_{ \pm}}{C_{0}} \frac{\partial C_{0}}{\partial z} \mp \Lambda_{ \pm} \frac{e}{k T} \frac{\partial \phi(r, z)}{\partial z}
$$

With the above relation and the Einstein relation $\mu / D=e / k T$, Eq.S26 is further simplified as

$$
\left(-D_{+} \frac{e}{k T} \frac{\partial V_{0}}{\partial z} \Lambda_{+}-D_{+} \Lambda_{+} \frac{\partial \ln C_{0}}{\partial z}\right)-\left(D_{-} \frac{e}{k T} \frac{\partial V_{0}}{\partial z} \Lambda_{-}-D_{-} \Lambda_{-} \frac{\partial \ln C_{0}}{\partial z}\right)=0
$$

It becomes Eq.14 in the maincontext after some straightforward algebra:

$$
\frac{\partial V_{0}}{\partial z}=-\frac{k T}{e} \frac{D_{+} \Lambda_{+}-D_{-} \Lambda_{-}}{D_{+} \Lambda_{+}+D_{-} \Lambda_{-}} \frac{\partial \ln C_{0}}{\partial z}
$$

For exclusion potential, we neglect the difference of diffusion coefficients between cations and anions $\left(D_{+} \approx D_{-}\right)$and arrive at the following expression for exclusion potential inside the monovalent systems is deduced:

$$
\frac{\partial V_{\sigma}(z)}{\partial z}=-E_{z}(z)=-\frac{\Lambda_{+}-\Lambda_{-}}{\Lambda_{+}+\Lambda_{-}} \frac{k T}{e} \frac{\partial \ln C_{0}}{\partial z}
$$

On the other hand, for the diffusion potential we neglect the counterions induced by the wall surface charges so that

$$
\begin{aligned}
& \Lambda_{ \pm}(z)=\pi R^{2} C_{0} \\
& \frac{\partial \Lambda_{ \pm}}{\partial z}=\pi R^{2} \frac{\partial C_{0}}{\partial z}
\end{aligned}
$$

In this way $V_{D}$ for monovalent system is derived from $\mathrm{S} 26$ :

$$
\frac{\partial V_{D}(z)}{\partial z}=-E_{z}(z)=-\frac{D_{+}-D_{-}}{D_{+}+D_{-}} \frac{k T}{e} \frac{\partial \ln C_{0}}{\partial z}
$$

For divalent ions, the expressions of self-built exclusion and diffusion voltages can be derived in a similar manner. Results are shown in the main context. 


\section{S5 Equation for nanofluidics under salt gradient}

\section{S5.1 Simplified view}

As we mentioned previously, due to the restriction by the nanochannel wall, the channel-radial motion of liquid is zero $\left(u_{r}=0\right)$. Therefore, the channel-axial component of the fluidics $u_{z}$ is determined from Navier-Stokes equations to be as follows

$$
\begin{gathered}
\frac{\partial u_{z}}{\partial z}=0 \\
\eta \frac{1}{r} \frac{\partial}{\partial r}\left(r \frac{\partial}{\partial r} u_{z}\right)-\frac{\partial p}{\partial z}+E_{z} e \sum_{i} z_{i} n_{i}=0
\end{gathered}
$$

At first glance, the above equations show that there are both hydrodynamic pressure and electrical body force to stimulate the liquid motion. However, we derive from the above equations the following relation

$$
\frac{\partial\left(-\frac{\partial p}{\partial z}+E_{z} e \sum_{i} z_{i} n_{i}\right)}{\partial z}=0
$$

It indicates that although the axial component of electrical body force $\vec{f}_{e} \cdot \vec{e}_{z}$ is not constant along the axis, the hydrodynamic pressure would adapt itself to make the overall force on the liquid to be axis-invariant. By integrating Eq.S36 over the axis and noticing that there is no pressure added from outside $\left(\left.p\right|_{z=-L / 2}=\left.p\right|_{z=L / 2}\right)$ we arrive at the following expression

$$
\eta \frac{1}{r} \frac{\partial}{\partial r}\left(r \frac{\partial}{\partial r} u_{z}\right)=-\frac{1}{L} \int_{-L / 2}^{L / 2} E_{z} e \sum_{i} z_{i} n_{i} d z
$$

Furthermore, for the nanoslit system used in the experiments [3] the above equation is rewritten in Descartes coordinate as follows:

$$
\eta \frac{\partial^{2}}{\partial y^{2}} u_{z}=-\frac{1}{L} \int_{-L / 2}^{L / 2} E_{z} e \sum_{i} z_{i} n_{i} d z
$$

\section{S5.2 Strict derivation}

The above derivation shows a simplified analysis of the nanofluidic system. Actually, a stricter derivation has to take into account the channel-radial component of the fluidics:

$$
-\frac{\partial p}{\partial r}-e \sum_{i} z_{i} n_{i} \frac{\partial \phi(r, z)}{\partial r}=0
$$

In the above, the restriction of no channel-radial fluidic speed $\left(u_{r}=0\right)$ has been used. By using the Boltzmann relation Eq.S3 we derive

$$
\frac{\partial \phi(r, z)}{\partial r}=-\frac{k T}{z_{i} e} \frac{1}{n_{i}} \frac{\partial n_{i}}{\partial r}
$$


Therefore Eq.S40 is further simplified as

$$
-\frac{\partial p}{\partial r}+k T \sum_{i} \frac{\partial n_{i}}{\partial r}=0
$$

Once again we separate the pressure into two parts:

$$
p(r, z)=p_{0}(z)+p_{\sigma}(r, z)
$$

The first term $\left.p_{0}\right|_{z= \pm L / 2}$ on the right represents the applied mechanical pressure from outside, and the second $p_{\sigma}(r, z)$ is induced by the channel-wall surface charge effect. Hence, we arrive at the following expression by using the above two equations:

$$
p_{\sigma}(r, z)=k T \sum_{i} n_{i}
$$

The above term is the so-called osmotic pressure conventionally. We are going to demonstrate that it would be offset by the charge effect. Since the pressure is divided into two components, Eq.S36 is then rewritten as

$$
\eta \frac{1}{r} \frac{\partial}{\partial r}\left(r \frac{\partial}{\partial r} u_{z}\right)-\frac{\partial p_{0}}{\partial z}-e \sum_{i} z_{i} n_{i} \frac{\partial V_{0}}{\partial z}-\frac{\partial p_{\sigma}(r, z)}{\partial z}-e \sum_{i} z_{i} n_{i} \frac{\partial \phi(r, z)}{\partial z}=0
$$

We remind that by utilizing the expression for $p_{\sigma}$, the fourth and five terms are counteracted. Therefore, Navier equation for channel-axial motion is deduced

$$
\eta \frac{1}{r} \frac{\partial}{\partial r}\left(r \frac{\partial}{\partial r} u_{z}\right)-\frac{\partial p_{0}}{\partial z}-e \sum_{i} z_{i} n_{i} \frac{\partial V_{0}}{\partial z}=0
$$

And then the fluidic kinetic equation shown in the main context is arrived at according to previous derivation.

\section{S6 The role of diffusio-osmosis}

It was proposed that there would be a diffusio-osmotic pressure induced by the salt concentration gradient within the electrical double layers $[1,6]$ :

$$
-\frac{\partial p_{D O}}{\partial z}=-k T \frac{\partial C_{0}}{\partial z}
$$

The diffusio-osmotic flow is then evaluated by

$$
\eta \frac{1}{r} \frac{\partial}{\partial r}\left(r \frac{\partial}{\partial r} u_{z}\right)=\left\{\begin{array}{cc}
k T \int_{-L / 2}^{L / 2} \frac{\partial C_{0}}{\partial z} d z & \left(R-\lambda_{D}<r \leq R\right) \\
0 & \left(r \leq R-\lambda_{D}\right)
\end{array}\right.
$$

We remind that $\lambda_{D}(z)$ is not a constant along $z$-direction due to the salt concentration gradient within the nanochannel. 
The calculated averaged fluid speed $\bar{u}_{z}$ as a function of the imposed salt concentration $C_{\max }$ at one side is plotted with black line in Fig.S6. As a comparison, the experimentally reported flow rate is also demonstrated by blue line with round symbols. The calculation indicates a monotone increasing reverse osmosis with the imposed salt gradient, which obviously does not agree with the 4-stage changes of fluid observed in the experiments [9] (Fig2b shown in the main context). Moreover, the magnitude of $\bar{u}_{z}$ boosted by the diffusio-osmotic pressure could reach $120 \mathrm{~mm} / \mathrm{s}$ at $C_{\max }=1 \mathrm{M}$, which is $3-4$ orders larger than that by the exclusion-diffusion potentials (as demonstrated in Fig.2b of the main context). In other words, when taking the diffusio-osmotic pressure into account, the exclusion-diffusion potentials proposed by us would become completely negligible, while on the other hand such results do not agree with the experiments [9] at all.

\section{S7 DNA capture by electrical field}

At the capture radius, the magnitude of electrophoretic flux is the same as that of diffusion one [7]:

$$
D \frac{C_{D N A}}{r^{*}}=\mu E\left(r^{*}\right) C_{D N A}
$$

where $D$ and $\mu$ are the diffusion coefficient and electrical mobility of DNA coils. By resorting to Eq.S8 the capture radius is obtained as

$$
r^{*}=\frac{\mu R^{2}}{2 D L}(\Delta V)_{p}
$$

The diffusion-limited DNA capture rate $\gamma$ is then given by

$$
\gamma=2 \pi r^{* 2} D \frac{C_{D N A}}{r^{*}}=\frac{\mu \pi R^{2} C_{D N A}}{L+\pi R / 2} V_{z}
$$

\section{S8 Exclusive electrical field in II region}

$$
\begin{gathered}
\left.C_{0}(z)\right|_{z=L / 2}=\frac{\pi R / 2}{2 L+\pi R} C_{\max }+\frac{2 L+\pi R / 2}{2 L+\pi R} C_{\min } \\
\left.\left(E_{z}\right)_{\sigma}\right|_{z=L / 2} \approx \frac{2 \sigma_{w}}{R N_{A} e} \frac{k T}{e} \frac{1}{\left(\frac{\pi R / 2}{2 L+\pi R} C_{\max }+\frac{2 L+\pi R / 2}{2 L+\pi R} C_{\min }\right)^{2}} \frac{C_{\max }-C_{\min }}{L+\pi R / 2}
\end{gathered}
$$

\section{S9 Fitting LiCl concentration gradient experi- ments}

Fig.S5 plots our simulation of self-built electrical potential $\Delta V$ and averaged fluid velocity $\bar{u}_{z}$ in the $\mathrm{LiCl}$ concentration biased nanopore systems. We try different values of pore-wall surface charge density $\sigma_{w}$ and demonstrate in Fig.S5a 
and $\mathrm{b}$ the results. We find that the larger $\sigma_{w}$, the stronger the exclusion effect. By setting $\sigma_{w}=-0.8 \mathrm{mC} / \mathrm{m}^{2}$, the calculation results match the experimental observation (blue rhombuses) the best.

Another issue is the actual concentration of electrolyte in the dilute chamber. In the experiment, $C_{m i n}=0$ at the beginning. However, as the ions diffused along the concentration gradient the final quantity of $C_{\min }$ was a positive value. Since we approximate the real situation with a static model, we have to determine the time-averaged value of $C_{m i n}$. We try $C_{m i n}=0.1 \mathrm{mM}, 1 \mathrm{mM}$ and 0.01 $\mathrm{mM}$ as seen in Fig.S5c and d. It indicates that by setting $C_{\min }=0.1 \mathrm{mM}$ the simulation results match best with the experiments.

\section{References}

[1] J L Anderson. "Colloid Transport by Interfacial Forces". In: Annu. Rev. Fluid Mech. 21.1 (1989), pp. 61-99. DOI: 10 .1146/annurev .fl . 21 . 010189 . 000425. eprint: http : / / dx . doi . org/10 . 1146/annurev . fl . 21.010189.000425. URL: http://dx.doi.org/10.1146/annurev.fl.21. 010189.000425.

[2] J. E. Hall. "Access Resistance of a Small Circular Pore". In: J. Gen. Physiol. 66 (1975), pp. 531-532.

[3] Sylvia Xin Li et al. "Direct Observation of Charge Inversion in Divalent Nanofluidic Devices". In: Nano Lett. 15.8 (2015), pp. 5046-5051. DOI: 10. 1021 /acs . nanolett . 5b01115. eprint: http://dx . doi .org/10 . 1021/ acs . nanolett . 5b01115. URL: http : / / dx . doi . org/10 . $1021 /$ acs . nanolett.5b01115.

[4] Kurt H. Meyer and J.-F. Sievers. "La perméabilité des membranes I. Théorie de la perméabilité ionique". In: Helv. Chim. Acta 19.1 (1936), pp. 649-664. ISSN: 1522-2675. DOI: 10.1002/hlca.19360190199. URL: http://dx.doi. org/10.1002/hlca.19360190199.

[5] Kurt H. Meyer and J.-F. Sievers. "La perméabilité des membranes. II. Essais avec des membranes sélectives artificielles". In: Helv. Chim. Acta 19.1 (1936), pp. 665-677. ISSN: 1522-2675. DOI: 10.1002/hlca.193601901100. URL: http://dx.doi.org/10.1002/hlca.193601901100.

[6] Alessandro Siria et al. "Giant Osmotic Energy Conversion Measured in a Single Transmembrane Boron Nitride Nanotube". In: Nature 494 (2013), pp. $455-458$.

[7] Meni Wanunu et al. "Electrostatic Focusing of Unlabeled DNA into Nanoscale Pores using a Salt Gradient". In: Nat. Nanotechnol. 5 (2010), pp. 160-165.

[8] G.B. Westermann-Clark and C.C. Christoforou. "The Exclusion-Diffusion Potential in Charged Porous Membranes". In: J. Electroanal. Chem. Interfac. 198.2 (1986), pp. 213 -231. ISSN: 0022-0728. DOI: http://dx.doi.org/ 10. 1016/0022-0728(86) 90001-X. URL: http : //www . sciencedirect . com/science/article/pii/002207288690001X. 
[9] Meng Yang et al. "Anomalous Effects of Water Flow through Charged Nanochannel Membranes". In: RSC Adv. 4 (51 2014), pp. 26729-26737. DOI: 10.1039/C4RA02856B. URL: http://dx.doi.org/10.1039/C4RA02856B. 


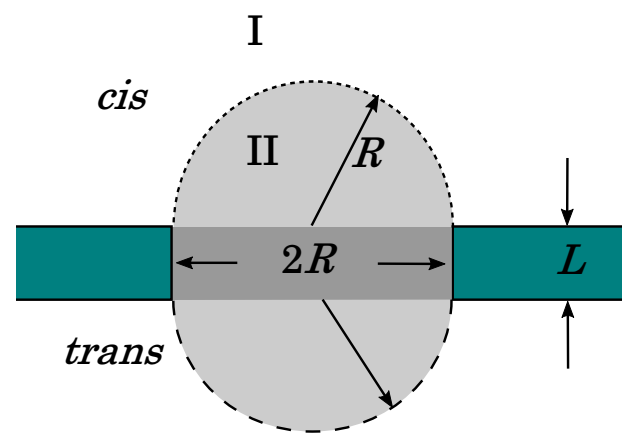

Figure S1: Schematic view of nanopore system. The cis/trans chamber is divided into two parts: I and II. 


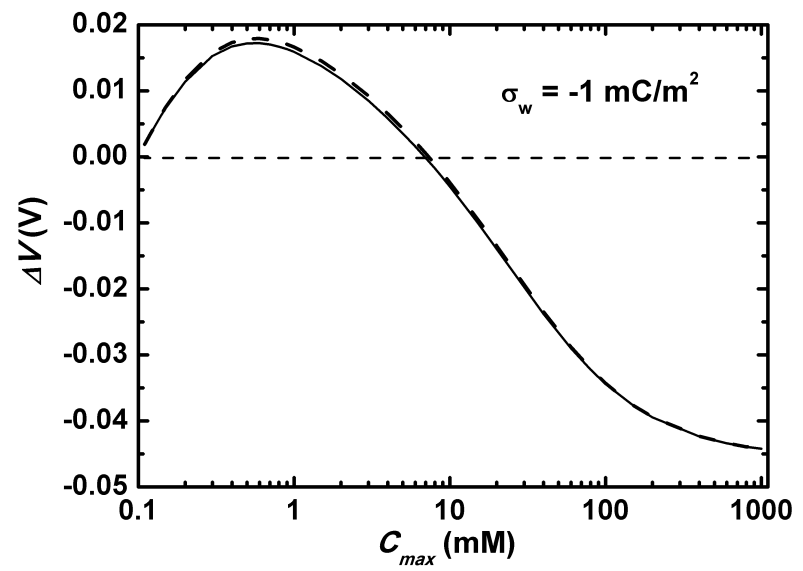

Figure S2: The calculated self-built voltage $\Delta V$ as a function of the imposed LiCl gradients $C_{\max }$ in a $R=50 \mathrm{~nm}$ and $L=6 \mu \mathrm{m}$ nanochannel. Here all parameters are the same as those in Fig.2 of the main context. Real lines show results by our model shown in the main context, while dash lines are based on Teorell-Meyer-Sievers model. 

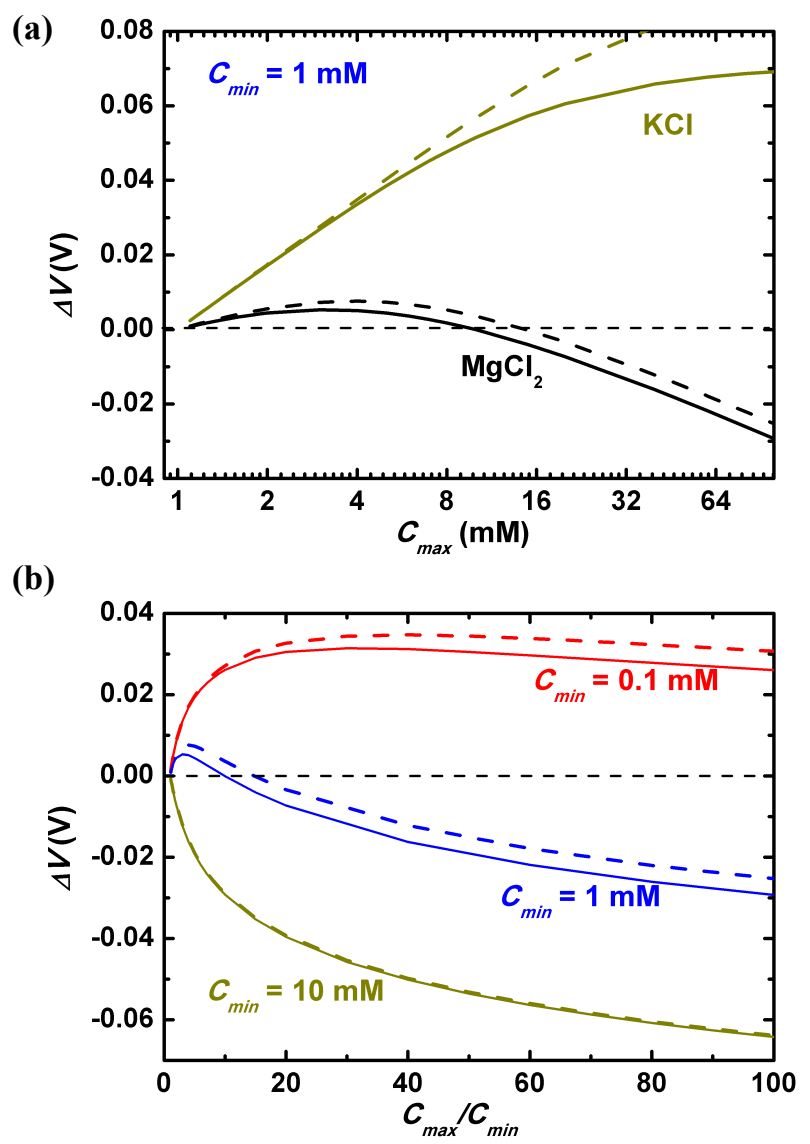

Figure S3: The calculated self-built voltage $\Delta V$ as a function of the imposed $\mathrm{MgCl}_{2}$ (a) and $\mathrm{KCl}$ (b) gradients $C_{\max } / C_{\min }$ in a $Y=20 \mathrm{~nm}$ and $L=116$ $\mu \mathrm{m}$ nanoslit. Here all parameters are the same as those in Fig.4 of the main context. Real lines show results by our model shown in the main context, while dash lines are based on Teorell-Meyer-Sievers model. 


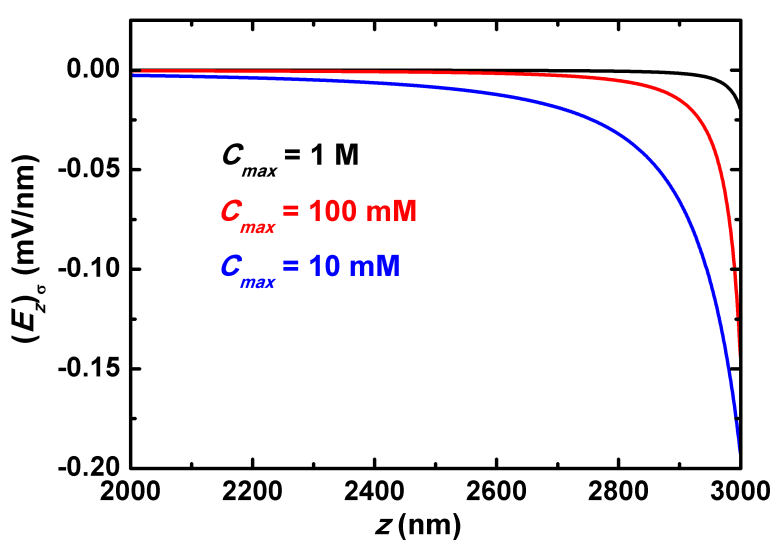

Figure S4: The calculated exclusive electrical field $\left.\left(E_{z}\right)\right|_{\sigma}$ along the channel axis under various $C_{\max }$. 

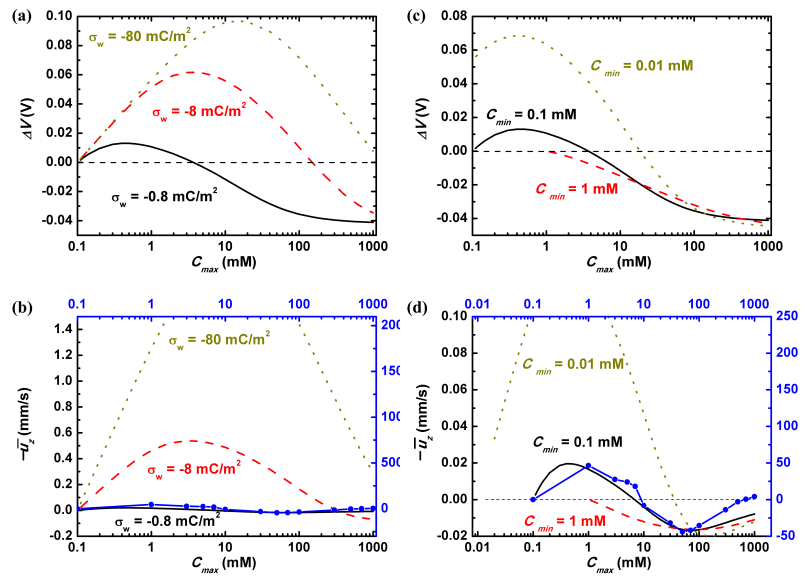

Figure S5: The calculated electrical potential $\Delta V$ ((a) and (c)) and the averaged fluid velocity $-\bar{u}_{z}((\mathrm{~b})$ and (d)) as functions of the larger salt concentration $C_{\max }$ at one side. In (a) and (b): The black, red and dark-yellow lines represent $\sigma_{w}=-0.8,-8$ and $80 \mathrm{mC} / \mathrm{m}^{2}$ respectively. In (c) and (d): The black, red and dark-yellow lines represent $C_{\min }=-0.1,1$ and $0.01 \mathrm{mM}$ respectively; the blue lines with round symbols plot the experimental data. 


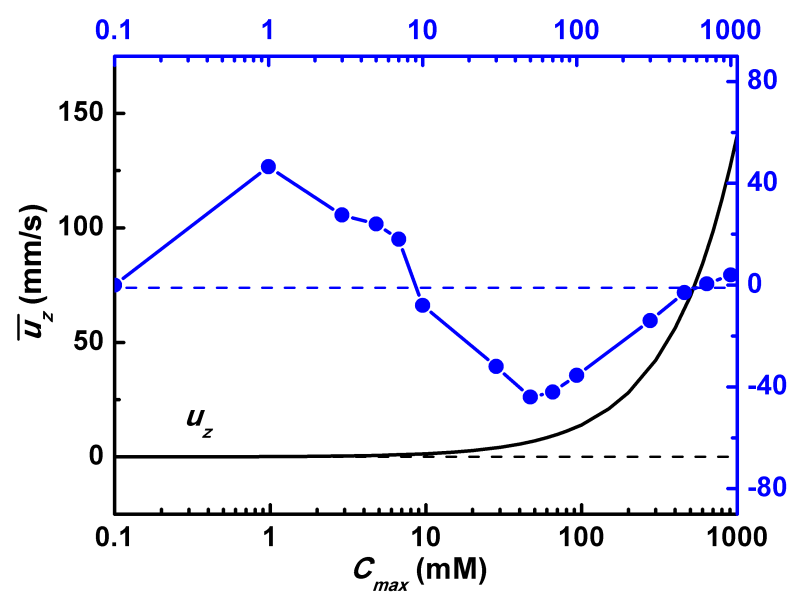

Figure S6: Left: The calculated averaged fluid speed $\bar{u}_{z}$ as a function of the imposed salt concentration $C_{\max }$ on one side when diffusio-osmotic pressure is the driving force. Right: the experimentally reported flow rate as a function of $C_{\max }$ [9]. $C_{\min }$ is set to be $0.1 \mathrm{mM}$, while other parameters are from the experiments: $R=50 \mathrm{~nm}$ and $L=6 \mu \mathrm{m}$. 